resections. Although higher, the increase in the overall $\mathrm{R} 0$ resection margins $(28.6 \%$ vs $36.3 \%, p=0.375)$ and the posterior resection margins $(42.9 \%$ vs $36.3 \%, p=0.481)$ didn't meet significance. The mean lymph node yield was significantly increased (19 vs 27, $\mathrm{p}<0.05)$ but there was no significant difference in the post-operative morbidity and mortality rates between the two groups. The actuarial 1 and 2 year survival was $72 \%$ and $49.4 \%$ for earlier resections and $71.8 \%$ and $56.8 \%$ for Artery first PPPD respectively. The 1 and 2 year disease free survival was $65.2 \%$ and $47 \%$ for earlier resections and $67.6 \%$ and $64.4 \%$ for Artery first PPPD.

Conclusion Artery first resection enhances the clearance at the SMA (a common site for R1 resection) with increased lymph node yield. The 2-year follow-up with Artery first PPPD shows a trend towards improving overall and disease free survival.

Competing interests None declared.

\section{OC-134 INTRA-ABDOMINAL METASTATIC NEUROENDOCRINE TUMOURS: HOW FEASIBLE IS THE LAPAROSCOPIC APPROACH TO LIVER RESECTIONS?}

doi:10.1136/gutjnl-2012-302514a.134

L M Edwards,* M J White, S 0 Cawich, M Abu Hilal, C Frola, N W Pearce. Department of Surgery, University Hospital Southampton NHS Foundation Trust, Southampton, UK

Introduction Approach to liver metastases in neuroendocrine tumours (NETs) significantly impacts on 5-year survival. A symptom based approach yields 5 -year survival of $30 \%$ compared to $>60 \%$ with multi-modal cytoreduction. ${ }^{1}$ At our NET tertiary referral centre patients are managed in line with the European Neuroendocrine Tumour Society guidelines, ${ }^{2}$ but with greater emphasis on multimodal cytoreduction for patients with unfavourable patterns of metastatic disease. We currently perform $60 \%$ of liver resections laparoscopically: over 300 procedures from 2003 to 2011. No existing study describes laparoscopic liver resections in patients with NETs. This study evaluates the feasibility and efficacy of the laparoscopic approach to liver resections in NETs in our tertiary referral unit.

Methods All patients assessed for intra-abdominal NETs from April 2005 to January 2012 were prospectively registered on a database. Patients undergoing laparoscopic liver resection were identified. Demographic, peri-operative and survival data were analysed using SPSS V.12.0. Severity of operative and post-operative morbidity was graded using the Clavien system.

Results The NET MDT assessed 239 patients. 99 patients had liver metastases; 58 underwent liver resection. 29 laparoscopic liver resections were performed on 27 patients (12F:15M), median age 63 (41-78). 11 major; eight minor and 10 multiple resections were performed. Intent of resection was curative $(n=4)$, cytoreductive $(n=19)$ or diagnostic/staging $(n=6)$. Peri-operative mortality was $0 \%$ with no cases of carcinoid crisis, bile leak or port site metastases. Metastatic resections with no primary resection $(n=27)$ had a complication rate of $11 \%(n=3)$ with no complication greater than grade IIIa. Median post-operative stay was 4 days (0-12). 24 patients are alive after median 32 months (9-64) followup post diagnosis and median 21 months (7-62) follow-up post surgery. 3 patients died of recurrence after median 25 months (20-30) follow-up post diagnosis and 20 months (16-25) follow-up post surgery.

Conclusion This is the first series to demonstrate laparoscopic liver resection for metastatic NETs can be performed with low morbidity and mortality by experienced surgeons in a tertiary referral unit.

Competing interests None declared.

\section{REFERENCES}

1. Saxena A, Chua T, Sarkar A, et al. Progression and survival results after radial hepatic metastectomy of indolent advanced neuroendocrine neoplasms (NENs) supports and aggressive surgical approach. Surgery 2011;149:209-20.

2. Steinmuller $\mathbf{T}$, Kianmanes $\mathrm{R}$, Falconi $\mathrm{M}$, et al. Consensus guidelines for the management of patients with liver metastases from digestive (neuro)endocrine tumors: foregut, midgut, hindgut and unknown primary. Neuro-endocrinology 2008;87:47-62.

\section{OC-135 LIVER RESECTION FOR COLORECTAL METASTASES: IS THERE VARIATION IN REFERRAL PRACTICE BETWEEN COLORECTAL MDTS IN A CANCER NETWORK? A PROSPECTIVE STUDY}

doi:10.1136/gutjnl-2012-302514a.135

${ }^{1} \mathrm{~A}$ Young, ${ }^{*}{ }^{1} \mathrm{R}$ Adair, ${ }^{2} \mathrm{~A}$ Culverwell, ${ }^{3} \mathrm{E}$ Morris, ${ }^{2} \mathrm{~A}$ Guthrie, ${ }^{2}$ Botterill, ${ }^{2} \mathrm{G}$ Toogood, ${ }^{2} \mathrm{P}$ Lodge, ${ }^{2} \mathrm{R}$ Prasad. ${ }^{1}$ Hepatobiliary Surgery; ${ }^{2}$ St James's University Hospital; ${ }^{3}$ Nycris, Leeds University, Leeds, UK

Introduction Half of all patients with colorectal cancer (CRC) develop liver metastases (LM). Liver resection offers the greatest survival benefit in suitable patients with 5 - and 10-year survival rates of $30 \%-58 \%$ and $25 \%$ respectively. There remains great variability in access to liver resection in the UK. We sought to investigate this within a cancer network known to already have a high rate of resection of CRCLM

Methods A prospective study of all patients presenting with new CRCLM in a cancer network was undertaken over a 12-month period. A study proforma was approved by the network to assess management decisions for all patients with CRCLM discussed at the seven CRC multidisciplinary team (MDT) meetings. Data were retrospectively collected on patients with CRCLM who did not have the proforma completed. Outcomes for all patients referred to the tertiary liver MDT were recorded and collated. Subsequently radiology of patients deemed inoperable at the CRC MDTs was reviewed by the liver MDT.

Results In total 631 patients with new CRCLM were assessed at CRC MDT meetings. Study forms were completed for 241 (38\%). $66 \%$ had synchronous disease and $42 \%$ unilobar disease. In all, $27 \%$ of patients were referred to the liver MDT for consideration of resection while $17 \%$ were deemed unfit, $2 \%$ refused referral, $17 \%$ gave no reason and $64 \%$ were thought inoperable. In the liver MDT, 142 new patients were discussed from this network. 74\% of patients reviewed were deemed suitable for further curative treatment; $52 \%$ resected and $22 \%$ neoadjuvant chemotherapy with a view to resection with a further $1 \%$ receiving ablation. Radiology for the patients considered fit but inoperable at CRC MDTs was retrospectively reviewed by a hepatobiliary radiologist and Liver surgeon and demonstrated a further $29 \%$ of patients were deemed operable and $15 \%$ of patients had equivocal imaging and warranted further investigation. Applied across the cancer network, this suggests that if all suitable patients were referred to the liver MDT, referral rates would increase by $142 \%$.

Conclusion Despite increased resection rates for CRCLM in the last decade, this prospective study shows a significant number of patients with potentially resectable disease are still not assessed in a specialist MDT. Improved referral rates may improve resection rates and improve overall outcomes for CRC patients. Extrapolation of these figures across the UK suggests improvements in referral practice of patients with CRCLM without any change in resection practice or post-resection outcomes could increase the number of patients with CRC in the UK alive at 5 years by $5 \%$.

Competing interests None declared. 